\title{
Role of spin current in magnetic relaxations of metallic multilayer films
}

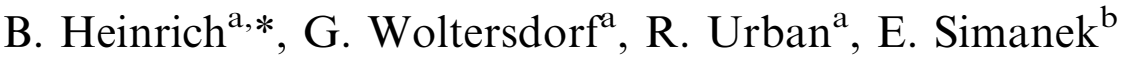 \\ a Physics Department, Simon Fraser University, Burnaby, BC, Canada V5A $1 S 6$ \\ ${ }^{\mathrm{b}} 6255$ Charing Lane, Cambria, CA 93428, USA
}

\begin{abstract}
In our recent studies the relaxation processes were investigated by ferromagnetic resonance (FMR) using magnetic single, $\mathrm{Au} / \mathrm{Fe} / \mathrm{GaAs}\left(\begin{array}{ll}0 & 0\end{array}\right)$, and double layer, $\mathrm{Au} / \mathrm{Fe} / \mathrm{Au} / \mathrm{Fe} / \mathrm{GaAs}\left(\begin{array}{ll}0 & 0\end{array}\right)$, structures prepared by molecular beam epitaxy (MBE). Single and double layer structures were grown on $(4 \times 6)$ reconstructed $\operatorname{GaAs}(001)$ templates. The samples have high crystalline quality with the magnetic properties corresponding to bulk $\mathrm{Fe}$, only modified by interface anisotropies. The magnetic relaxation in these structures is well described by Gilbert damping with no extrinsic contributions to the FMR line width. These structures provide an excellent opportunity to investigate non-local damping which is caused by spin transport across a non-magnetic spacer. In the double layer structures thin Fe layers were separated from a second thick Fe layer of 40 monolayer (ML) thickness by a $40 \mathrm{ML}$ thick $\mathrm{Au}(001)$ spacer. The main result of these studies is that the ultrathin Fe films in magnetic double layers acquire an additional Gilbert damping. The additional FMR line width followed an inverse dependence on the Fe film thickness which is expected for interface damping. The spin pump model will be discussed and it will be shown that it is closely related to the dynamic part of interlayer exchange coupling.
\end{abstract}

(C) 2002 Elsevier Science B.V. All rights reserved.

Keywords: Spin transport; Magnetic relaxation; Magnetic multilayers; MBE; FMR

\section{Introduction}

The development of magnetic devices operating at high frequencies requires a thorough understanding of the mechanisms responsible for magnetic relaxation. Spin dynamics in the classical limit can be described by the Landau-Lifshitz-Gilbert equation of motion $[1,2]$

$\frac{1}{\gamma} \frac{\partial \vec{M}}{\partial t}=-\left[\vec{M} \times \vec{H}_{\mathrm{eff}}\right]+\frac{G}{\gamma^{2} M_{\mathrm{s}}^{2}}\left[\vec{M} \times \frac{\partial \vec{M}}{\partial t}\right]$

where the first term on right-hand side represents the precessional torque and the second term represents the relaxation term which is expressed by the well-known Gilbert damping torque [2]. $H_{\text {eff }}$ represents an effective field which is given by $\vec{H}_{\text {eff }}=-\partial E / \partial \vec{M}$, where $E$ is the Gibbs free energy [3]. Recently, we showed that

\footnotetext{
*Corresponding author. Fax: + 1-604 2913-592.

E-mail address: bheinric@sfu.ca (B. Heinrich).
}

magnetic multilayers exhibit non-local Gilbert damping [4]. These results supported Berger's prediction of nonlocal damping in a $\mathrm{FM} / \mathrm{NM} / \mathrm{FM}$ structure, where $\mathrm{FM}$ and NM stand for a ferromagnetic and a normal metal layers. Berger showed [5] that the transfer of angular momentum between FM layers results in an additional ferromagnetic resonance (FMR) line width, $\Delta H_{\text {add }}$, which is given by

$\Delta H_{\text {add }}=\operatorname{coeff}(\Delta \mu+\hbar \omega)$,

where $\Delta \mu=\Delta \mu^{\uparrow}-\Delta \mu^{\downarrow}$ is the difference in the spin up and spin down Fermi level shifts, and $\omega$ is the microwave angular frequency. $\Delta \mu$ can be controlled by an applied DC current flowing perpendicular to the film surface [5]. In the absence of an applied DC current $\Delta \mu$ is only negative, and for large precessional amplitudes it saturates at $-\hbar \omega$ [6]. Berger showed that the s-d exchange interaction between a spin wave and itinerant electrons in magnetic multilayers takes a new form in the vicinity of the interface between the NM spacer and 
precessing ferromagnetic layer. It leads to an interface Gilbert damping. The purpose of this paper is to briefly review our recent experimental results of non-local damping in magnetic multilayers, and compare them with theoretical models.

\section{Magnetic multilayers and experimental results}

The preparation of the ultrathin film structures was carried out by means of molecular beam epitaxy (MBE). Semi-insulating epi-ready $\operatorname{GaAs}\left(\begin{array}{lll}0 & 0 & 1\end{array}\right)$ wafers were used as templates for the growth of metallic structures. The GaAs substrates were heated to approximately $500^{\circ} \mathrm{C}$ in order to desorb contaminants [7]. Residual oxides were removed using a low-energy $\mathrm{Ar}^{+}$bombardment $(0.6 \mathrm{keV})$ under grazing incidence. Substrates were rotated around their normal during the sputtering. Subsequent annealing up to $600^{\circ} \mathrm{C}$ was monitored using reflection high-energy electron diffraction (RHEED) until a well ordered $(4 \times 6)$ reconstruction appeared. Our scanning tunnelling microscopy studies [8] showed that the $4 \times 6$ reconstruction consists of $(1 \times 6)$ and $(4 \times 2)$ domains. The $(1 \times 6)$ domain is As rich, while the $(4 \times 2)$ domain is $\mathrm{Ga}$ rich.

Iron films were deposited at room temperature (RT) from a thermal source at a base pressure of less then $2 \times 10^{-10}$ Torr and the deposition rate was $\sim 1$ monolayer $(\mathrm{ML}) / \mathrm{min}$. The film thickness was monitored by a quartz crystal microbalance and by means of RHEED intensity oscillations. During the growth, approximately 0.6 ML of As segregates on top of the Fe film [7]. When additional metallic layers were deposited, the same amount of As was observed by X-ray photoemission spectroscopy. The floating As atoms act as a surfactant; no As stays inside the deposited structure. The gold was evaporated at RT at the deposition rate of $\sim 1 \mathrm{ML} / \mathrm{min}$. RHEED oscillations were visible for up to 30 atomic layers. Due to the presence of surfactant As, the RHEED diffraction pattern of $\mathrm{Au}(001)$ showed a $2 \times 2$ reconstruction.

Single Fe ultrathin films with the thicknesses $d=8$, $11,16,21,31 \mathrm{ML}$ were grown directly on $\mathrm{GaAs}\left(\begin{array}{ll}0 & 0\end{array}\right)$.

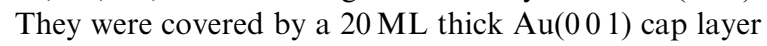
for protection in ambient conditions. The FMR measurements were used to determine the in-plane cubic and uniaxial magnetic anisotropies, $K_{1}$ and $K_{\mathrm{u}}$, and the effective demagnetizing field perpendicular to the film surface, $4 \pi M_{\text {eff }}$ as a function of thickness [7]. The magnetic anisotropies are well described by a linear dependence on $1 / d$, where $d$ is the Fe film thickness. The constant and linear terms represent the bulk and interface magnetic properties, respectively. The $\mathrm{Fe}$ ultrathin films grown on $\operatorname{GaAs}(001)$ and covered by gold have magnetic properties nearly equal to those in the bulk Fe, modified only by sharply defined interface anisotropies. $\quad 4 \pi M_{\mathrm{s}}=20.8 \mathrm{kOe}, \quad K_{\perp}^{\mathrm{s}}=0.9 \mathrm{ergs} / \mathrm{cm}^{2}$, $K_{1}=4.3 \times 10^{5} \mathrm{ergs} / \mathrm{cm}^{3}, \quad K_{1}^{\mathrm{s}}=-0.02 \mathrm{ergs} / \mathrm{cm}^{2}, \quad$ and $K_{\mathrm{u}}^{\mathrm{s}}=-0.07 \mathrm{ergs} / \mathrm{cm}^{2}$, where superscript "s" stands for the interface anisotropy.

The reproducible magnetic anisotropies and small FMR line widths provided an excellent opportunity for the investigation of non-local relaxation processes in multilayer films. The studies were carried out using the simplest multilayer structure which consists of only two magnetic layers separated by a non-magnetic spacer, usually referred to as a magnetic double layer.

The Fe films which were studied in the single layer structures were re-grown as one element of a double layer structure. For further reference they will be called F1 layers. They were separated from a thick Fe layer of $40 \mathrm{ML}$ thickness by a $40 \mathrm{ML}$ thick Au spacer. For further reference the thick layer will be called F2. The double layers were covered by $20 \mathrm{ML}$ of $\mathrm{Au}\left(\begin{array}{lll}0 & 0 & 1\end{array}\right)$ for protection in ambient conditions. The complete structure was $\mathrm{GaAs} / \mathrm{Fe}(8,11,16,21,31) / 40 \mathrm{Au} / 40 \mathrm{Fe} /$ $20 \mathrm{Au}(001)$, where the integers represent the number of ML. The interface magnetic anisotropies allowed us to separate the FMR field of the thin layers F1 from the thick layer F2 by a sufficient margin that in most cases the difference in FMR fields exceeds $5 \times$ their FMR line widths. Hence, the FMR measurement for F1 in double layers was carried out under such conditions that the magnetic moment of F2 was nearly static with no appreciable angle of precession. The main results are summarized in Fig. 1.

The FMR line width of $\mathrm{F} 1$ always increases in the presence of F2. The difference in the FMR line widths, $\Delta H_{\text {add }}$, between the double and single layer structures followed well the inverse dependence on the thin film thickness, $d$, see Fig. $1 \mathrm{~b}$. The overall $1 / d$ dependence of $\Delta H_{\text {add }}$ shows that the non-local Gilbert damping originates at the F1/NM interface. The linear dependence of $\Delta H_{\text {add }}$ on the microwave frequency, $f$, for parallel and perpendicular FMR is equally important. It means that the additional contribution to the FMR line width can be described by interface Gilbert damping. The additional Gilbert damping, $G_{\text {add }}$, for the $16 \mathrm{ML}$ thick film was found to be weakly dependent on the orientation of the magnetization with respect to the crystallographic directions, $G_{\text {add }}=1.2 \times 10^{8} \mathrm{~s}^{-1}$ along the cubic axis. Note that the non-local damping is an appreciable effect. It has a strength comparable to the intrinsic Gilbert damping in a single $\mathrm{Fe}$ film, $G=1.5 \times 10^{8} \mathrm{~s}^{-1}$. It is worthwhile of mentioning that the FMR line width in F1 in single layer structures was found only weakly dependent on the film thickness.

\section{Discussion of results}

The presence of a strict Gilbert damping in the parallel and perpendicular FMR measurements is not 

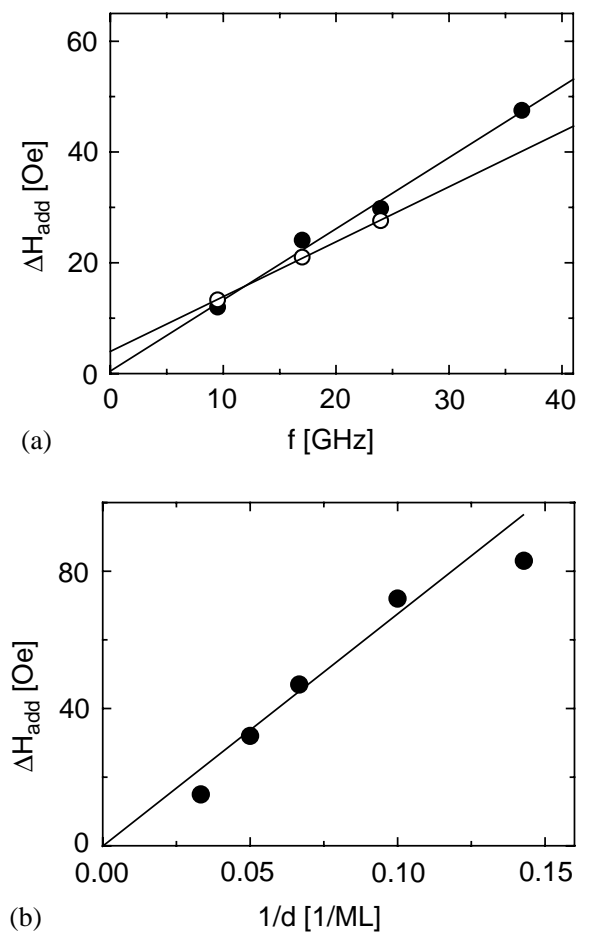

Fig. 1. (a) The additional FMR line width, $\Delta H_{\text {add }}=\Delta H_{\text {double }}-$ $\Delta H_{\text {single }}$, for the $16 \mathrm{ML}$ thin film in the magnetic double layer $\mathrm{GaAs} / 16 \mathrm{Fe} / 40 \mathrm{Au} / 40 \mathrm{Fe} / 20 \mathrm{Au}(001)$ as a function of the microwave frequency $f$. Filled circles $(\bullet)$ represent the in-plane FMR measurements (the saturation magnetization and applied external field parallel to the film plane), empty circles $(O)$ correspond to the perpendicular FMR measurements (the saturation magnetization and applied external field perpendicular to the film surface). The number of points for the perpendicular FMR measurements is limited by the available upper value of the external DC magnetic field. The FMR line width was determined by the HWHM (half-width at halfmaximum) of the imaginary part of the RF susceptibility, $\chi^{\prime \prime}$. (b) The additional FMR line width, $\Delta H_{\text {add }}$, along the Fe cubic axis as a function of $1 / d$ at $f=36 \mathrm{GHz}$. $d$ is the thickness of $\mathrm{F} 1$ films. The solid line is used to guide reader's eye.

easy to reconcile with Berger's model. Berger's theoretical model suggests that the interface damping $(\sim \hbar \omega)$ and the angular momentum transport by an applied DC current $(\sim \Delta \mu)$, see Eq. (2), belong to the same relaxation torque. In this case they should be a part of Slonczewski's torque for spin momentum transport [9]

$\vec{T}_{\mathrm{Sl}}=\operatorname{coef}(\Delta \mu+\hbar \omega) \frac{\vec{M}}{M_{\mathrm{S}}} \times \vec{c} \times \frac{\vec{M}}{M_{\mathrm{S}}}$,

where $\vec{c}$ is the direction of the stationary magnetization of the F2 layer, and coef is a common pre-factor. Eq. (3) results in Gilbert damping for the perpendicular configuration, but it leads to Bloch Blombergen-like damping for the parallel configuration with the relaxation time constant proportional to the microwave frequency $\omega$. For the parallel configuration the FMR line width would be proportional to $(\omega / \gamma)^{2} /(B+H)$. This has a non-linear dependence on the microwave frequency. Clearly, the mechanism of non-local damping has to be reformulated.

Recently Tserkovnyak et al. [10] showed that the interface damping can be generated by a spin current from a ferromagnet into adjacent NM layers. The spin current is generated by a precessing magnetization. The spin current was calculated by using Brouwer's scattering matrix [11] which evolves under a time dependent parameter (angle of precession). NM layers surrounding a magnetic layer were viewed as reservoirs in common thermal equilibrium. The calculations were carried out by assuming that the reservoirs acted as ideal spin sinks. This approximation is valid when the injected spin momentum into a normal metal decays or leaves the interface sufficiently fast to avoid the flow of spin current back into the ferromagnet.

The resulting spin current is

$\vec{j}_{\text {spin }}=\frac{\hbar}{4 \pi} A_{\mathrm{r}} \vec{m} \times \frac{\partial \vec{m}}{\partial t}$,

where $\vec{m}$ is the unit vector along the magnetic moment $\vec{M}$, and $A_{\mathrm{r}}$ is the scattering parameter

$A_{\mathrm{r}}=\sum_{m, n}\left(\left|r_{m, n}^{\uparrow}-r_{m, n}^{\downarrow}\right|^{2}+\left|t_{m, n}^{\uparrow}-t_{m, n}^{\downarrow}\right|^{2}\right)$,

where $r_{m, n}^{\uparrow \downarrow}$ are the reflection parameters for spin up and spin down electrons in the NM reservoirs, and $t_{m, n}^{\uparrow \downarrow}$ are the transmission parameters into the reservoirs. The index $n$ and $m$ in Eq. (5) labels the transverse modes $(\vec{k}$ wave vectors parallel to the interface) at the Fermi energy. Note that, in contrast to Berger's theory [5], the contribution to damping disappears in the absence of $\mathrm{s}-\mathrm{d}$ exchange splitting. The spin pump effect is not present in absence of a long-range ferromagnetic state. The spin current has the form of Gilbert damping, see Eq. (1). The Gilbert damping is given by conservation of the total spin momentum

$\vec{j}_{\text {spin }}-\frac{1}{\gamma} \frac{\partial \vec{M}_{\text {tot }}}{\partial t}=0$,

where $\vec{M}_{\text {tot }}$ is the total magnetic moment. After simple algebraical steps one obtains the expression for the dimensionless damping parameter

$\alpha=\frac{G}{\gamma} \frac{1}{M_{\mathrm{s}}}=g \mu_{\mathrm{B}} \frac{A_{r}}{4 \pi M_{\mathrm{S}}} \frac{1}{d}$,

where $d$ is the thickness of the ferromagnetic layer, and $A_{r}$ is now evaluated for a unit interfacial area. The inverse dependence of the Gilbert damping on the film thickness clearly testifies to its interfacial origin.

The layer F1 acts as a spin pump. Now another important point has to be answered: How is the generated spin current disposed? This answer can be found in the recent article by Stiles and Zangwill on 
"Anatomy of spin transfer torque" [12]. They showed that the transversal component of the spin current in a normal layer NM is entirely absorbed at the NM/FM interface. For small precessional angles the spin current $\vec{j}_{\text {spin }}$ is almost entirely transversal. It means that the NM/ F2 interface acts as an ideal spin sink, and provides an effective spin brake for F1. This assertion can be tested by investigating the FMR line width around an accidental crossover of the resonance fields for F1 and F2. The large in-plane uniaxial anisotropy in the thinner sample, F1, compared to that of the thicker sample, F2, created and an accidental crossover of the resonance fields. In this case the resonant field of F2 approached the resonant field of $F 1$. When they reached the same resonant field the RF magnetization components of F1 and F2 were parallel to each other. Each precessing magnetization created its own spin current which was pumped across the NM spacer. The electron mean free path in Au thick films is $38 \mathrm{~nm}$ [13], and consequently the spin transport even in a $40 \mathrm{ML}$ thick Au spacer was purely ballistic. At the same time the both interfaces F1/ $\mathrm{NM}$ and $\mathrm{NM} / \mathrm{F} 2$ acted as spin sinks. It follows that the net flow of the spin current through each interface was zero, and the additional FMR line width had to disappear. Indeed for the same resonance fields the FMR resonance became narrow and corresponded to

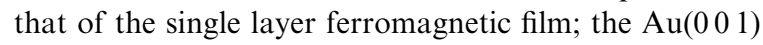
capping layer is much thinner than the spin diffusion length. The spin momentum accumulates in the $\mathrm{Au}(001)$ capping layer resulting in zero additional damping [10].

However, a word of caution is needed. The same resonant fields do not have to necessarily create the same spin current. A different elliptical polarization and intrinsic damping in $\mathrm{F} 1$ and $\mathrm{F} 2$ can result in different magnitudes of the transversal RF magnetization components. One can evaluate the appropriate terms $\vec{m} \times$ $\partial \vec{m} / \partial t$ for the 16 and $40 \mathrm{ML}$ thick films by using their magnetic properties. It turns out that the difference between these terms drops to $\frac{1}{40}$ and $\frac{1}{25}$ of their maximum values (corresponding to a large difference in the FMR fields) for the components along and perpendicular to the film surface, respectively. Clearly, in our case the net spin currents are nearly equal to zero at the crossover, $H_{\text {res, } 16}-H_{\text {res, } 40}=0$, and with that indeed the additional FMR line width disappeared as expected. This result greatly supports the concept of spin pump and spin sink in magnetic multilayers.

The spin pump model is a rather exotic theory to those who are used to magnetic studies. One would expect that there is a direct connection to a more common concept which is applicable to magnetic multilayers. The obvious choice is interlayer exchange coupling. The interlayer exchange interaction was so far treated only in static limit [14]. One would expect that its dynamic part can create magnetic damping.
A ferromagnetic sheet surrounded by a normal metal can be investigated by using a contact exchange interaction between the ferromagnetic spins and electrons in the normal metal. One can expand the linear response Kubo theory [15] for slow precessional motion using a linear approximation for a retarded magnetic moment

$\vec{S}(t-\tau) \cong \vec{S}(t)-\tau \frac{\partial \vec{S}}{\partial t}$,

where $\vec{S}(t)$ is the spin moment of the magnetic sheet at the instantaneous time $t$ and $\tau$ is the delayed time in the retarded response. It can be shown that one obtains Gilbert damping which for the same interaction is equivalent to that obtained using the spin current calculations [16]. The advantage of dynamic exchange coupling model is that it allows one to include electron correlation effects such as the Stoner enhancement factor $[17,18]$. In fact our recent results using $20 \mathrm{Au} /$ $40 \mathrm{Fe} / 40 \mathrm{Au} / 3 \mathrm{Pd} /[\mathrm{Fe} / \mathrm{Pd}]_{5} / 14 \mathrm{Fe} / \mathrm{GaAs}\left(\begin{array}{lll}0 & 0 & 1\end{array}\right) \quad$ samples showed a significant enhancement and strong four-fold in-plane anisotropy for the interface damping, see Fig. 2. The additional interface damping can be enhanced as much as by a factor of four along the cubic crystallographic axis. The metallic $\mathrm{Pd}$ is known to exhibit a strong Stoner enhancement in DC susceptibility. The results in Fig. 2 show that electron correlation effects in NM can be important.

One can also calculate a non-local damping by directly employing interlayer exchange coupling using a kinetic approach. Dynamics is accounted for by using a delayed response of electrons in the NM spacer in the presence of precessing magnetization. A similar approach was used by Kambersky [19] to calculate the intrinsic damping in bulk ferromagnets using the spinorbit interaction. The energy density which can be used to evaluate an effective field acting on the magnetic layer $\mathrm{F} 1$ is given by

$E_{\text {exch }}=\frac{1}{\Omega} \sum_{\vec{k}, \sigma} n_{\vec{k}, \sigma} \varepsilon_{\vec{k}, \sigma}$,

where $n_{\vec{k}, \sigma}$ and $\varepsilon_{\vec{k}, \sigma}$ are the occupation number and energy of electrons for the state described by the wave vector $\vec{k}$ and spin $\sigma$ participating in the interlayer exchange coupling. $\Omega=$ Area $d$ is the volume of the magnetic layer, F1.

The effective field that acts on the layer $F 1$ is given by

$\vec{H}_{\text {exch }}=-\frac{\partial E_{\text {exch }}}{\partial \vec{M}_{F 1}}=-\frac{1}{\Omega} \sum_{\vec{k}, \sigma} n_{\vec{k}, \sigma} \frac{\partial \varepsilon_{\vec{k}, \sigma}}{\partial \vec{M}_{F 1}}$,

where $\vec{M}_{F 1}$ is the magnetization vector of $\mathrm{F} 1$.

The energy of electrons is dependent on the instantaneous orientation of the magnetic moments, and consequently the electronic states keep redistributing 


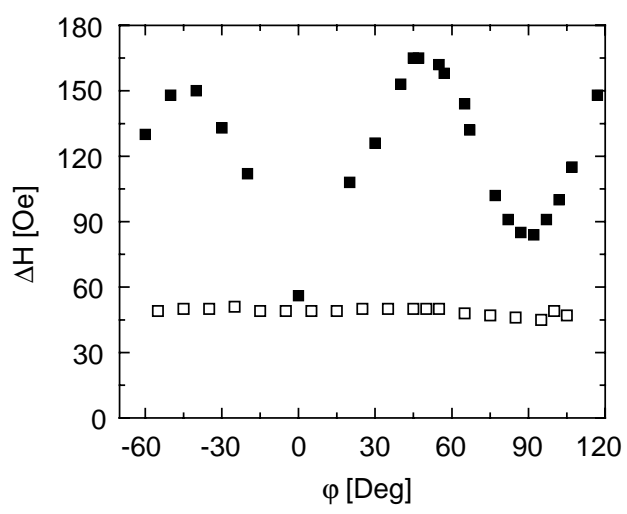

Fig. 2. The dependence of the FMR line width at $36 \mathrm{GHz}$ which was measured for a $14 \mathrm{Fe}[/ 1 \mathrm{Pd} / 1 \mathrm{Fe}]_{5}$ layer as a function of the angle $\phi$ between the external field and the in-plane crystallographic axis. The $14 \mathrm{Fe}[1 \mathrm{Pd} / 1 \mathrm{Fe}]_{5}$ layer is fully ferromagnetic and is a part of the $\mathrm{GaAs} / 14 \mathrm{Fe}[/ 1 \mathrm{Pd} / 1 \mathrm{Fe}]_{5} / 3 \mathrm{Pd} / 40 \mathrm{Au} /$ $40 \mathrm{Fe} / 20 \mathrm{Au}(001)$ structure. The normal metal spacer is composed of $3 \mathrm{Pd} / 40 \mathrm{Au}(001)$. Open square symbols $(\square)$ correspond to the single layer measurements using a $\mathrm{GaAs} /$ $14 \mathrm{Fe}[/ 1 \mathrm{Pd} / 1 \mathrm{Fe}]_{5} / 20 \mathrm{Au}(0001)$ structure, and filled square symbols ( $\mathbf{\square})$ correspond to the double layer measurements using a $\mathrm{GaAs} / 14 \mathrm{Fe}[/ 1 \mathrm{Pd} / 1 \mathrm{Fe}]_{5} / 3 \mathrm{Pd} / 40 \mathrm{Au} / 40 \mathrm{Fe} / 20 \mathrm{Au}(0001)$ structure. Note that at $\phi=0$ the FMR line width for the $14 \mathrm{Fe}[1 \mathrm{Pd} /$ $1 \mathrm{Fe}]_{5}$ layer is decreased down to the value which was observed for the single layer structure $\mathrm{GaAs} / 14 \mathrm{Fe}[/ 1 \mathrm{Pd} / 1 \mathrm{Fe}]_{5} /$ $20 \mathrm{Au}(001)$. The resonant fields in $\mathrm{GaAs} / 14 \mathrm{Fe}[/ 1 \mathrm{Pd} / 1 \mathrm{Fe}]_{5} /$ $3 \mathrm{Pd} / 40 \mathrm{Au} / 40 \mathrm{Fe} / 20 \mathrm{Au}(001)$ in this case were accidentally almost identical and that resulted in a negligible non-local damping. The FMR line width at $\phi=90^{\circ}$ for the $14 \mathrm{Fe}[/ 1 \mathrm{Pd} /$ $1 \mathrm{Fe}]_{5}$ film is narrower than that for $\phi=45^{\circ}$, but the corresponding resonant fields were far apart. The observed decrease in FMR line width around $\phi=90^{\circ}$ is a genuine anisotropic effect of the non-local damping in $\mathrm{GaAs} / 14 \mathrm{Fe} / / 1 \mathrm{Pd} /$ $1 \mathrm{Fe}]_{5} / 3 \mathrm{Pd} / 40 \mathrm{Au} / 40 \mathrm{Fe} / 20 \mathrm{Au}(0001)$ structures.

while the magnetization remains precessing. In the presence of a time dependent potential the Fermi surface changes in time; one often refers to this effect as "breathing Fermi surface".

However, this redistribution cannot be achieved instantaneously. There is a time delay which can be described by an effective relaxation time $\tau$. The "breathing Fermi surface" is driven by the exchange field of the precessing magnetic moment. This results in the exchange induced moment in the NM spacer (achieved by redistribution of the electron occupation numbers $n_{\vec{k}, \sigma}$ ). The time lag between the instantaneous exchange field and the induced moment in the spacer is described by the transverse spin relaxation time, $\tau=\tau_{\text {spin }}$. In the limit of a slow precessional motion the instantaneous electron distribution can be approximated by

$n_{\vec{k}, \sigma}(t)=n_{\vec{k}, \sigma, \vec{M}}(t)-\tau_{\text {spin }} \frac{\partial n_{\vec{k}, \sigma, \vec{M}}(t)}{\partial t}$, where $n_{\vec{k}, \sigma, \vec{M}}(t)$ is the static occupation number for the magnetic moment of the layer $\mathrm{F} 1$ with the magnetization $\vec{M}$. The first term in Eq. (11) would provide the static interlayer exchange coupling field, and the second term provides damping.

The effective damping field can be evaluated by using Eqs. (10) and (11)

$$
\begin{aligned}
& \vec{H}_{\text {damping }} \\
& =\tau_{\text {spin }} \frac{\sum_{\vec{k}, \sigma} \frac{\partial n_{\vec{k}, \sigma, \vec{M}}(t)}{\partial t} \frac{\partial \varepsilon_{\vec{k}, \sigma, \vec{M}}}{\partial \vec{M}}}{\text { Area }} \frac{1}{d} \\
& =\tau_{\text {spin }} \frac{\sum_{\vec{k}, \sigma} \frac{\partial n_{\vec{k}, \sigma, \vec{m}}}{\partial \varepsilon_{\vec{k}, \sigma, \vec{m}}}\left(\frac{\partial \varepsilon_{\vec{k}, \sigma, \vec{M}}}{\partial \vec{M}}\right)^{2}}{\text { Area }} \frac{1}{d} \frac{\partial \vec{M}}{\partial t} \\
& =\tau_{\text {spin }} \frac{\sum_{\vec{k}, \sigma} \delta\left(\varepsilon_{\vec{k}, \sigma, \vec{M}}-\varepsilon_{F}\right)\left(\frac{\partial \varepsilon_{\vec{k}, \sigma, \vec{M}}}{\partial \vec{M}}\right)^{2}}{\text { Area }} \frac{1}{d} \frac{\partial \vec{M}}{\partial t},
\end{aligned}
$$

where Area is the area of the film, and $d$ is the thickness of the ferromagnetic film. The effective damping field is proportional to the time derivative of the magnetic moment, this is a clear indication of Gilbert damping. Note also that in Eq. (12) the effective field scales inversely with film thickness, and therefore provides interface Gilbert damping. The electron energies $\varepsilon_{\vec{k}, \sigma, \vec{M}}$ can be calculated using Slonczewski's method [14]. The above model suggests that the non-local damping in magnetic multilayers can exhibit an oscillatory behavior with the spacer thickness. Our recent experiments have shown an evidence for oscillatory Gilbert damping.

\section{Conclusions}

We have shown that a non-local damping by the transfer of spin momentum can be realized in magnetic multilayer films. The effect can be significant. By a proper engineering of interfaces one can create magnetic damping which significantly surpasses that in single layers. Theoretical models were presented for non-local damping. It was shown that the non-local damping in magnetic multilayers is well described by a concept of spin pumps and spin sinks. This effect is directly related to dynamics of interlayer exchange coupling.

\section{References}

[1] L.D. Landau, E.M. Lifshitz, L.P. Pitaevski, Statistical Physics, 3rd Edition, part 2, Pergamon, Oxford, 1980.

[2] T.L. Gilbert, Phys. Rev. 100 (1955) 1243.

[3] B. Heinrich, J.F. Cochran, Adv. Phys. 42 (1993) 523.

[4] R. Urban, W. Woltersdorf, B. Heinrich, Phys. Rev. Lett. 87 (2001) 217204. 
[5] L. Berger, Phys. Rev. B 54 (1996) 9353.

[6] L. Berger, J. Appl. Phys. 90 (2001) 4632.

[7] T.L. Monchesky, B. Heinrich, R. Urban, K. Myrtle, M. Klaua, J. Kirschner, Phys. Rev. B 60 (1999) 10242.

[8] T. Monchesky, R. Urban, B. Heinrich, M. Klaua, J. Kirschner, J. Appl. Phys. 87 (2000) 5167.

[9] J.C. Slonczewski, J. Magn. Mag. Mater. 159 (1996) 1.

[10] Y. Tserkovnyak, A. Brataas, G.E.W. Bauer, Phys. Rev. Lett. 88 (2002) 117601.

[11] P.W. Brouwer, Phys. Rev. B 58 (1998) R10135.

[12] M.D. Stiles, A. Zangwill, Phys. Rev. B 66 (2002) 014407.
[13] A. Enders, T. Monchesky, K. Myrtle, R. Urban, B. Heinrich, J. Kirschner, X.G. Zhang, W.H. Butler, J. Appl. Phys. 89 (2001) 7110.

[14] J.C. Slonczewski, J. Magn. Magn. Mater. 126 (1993) 374.

[15] A.L. Fetter, J.D. Walecka, Quantum Theory of ManyParticle Systems, McGraw Hill, New York, 1971.

[16] E. Simanek, B. Heinrich, cond-mat/0207471.

[17] P.A. Wolff, Phys. Rev. 120 (1960) 814.

[18] T. Izuyama, D.J. Kim, R. Kubo, J. Phys. Soc. Jpn. 18 (1963) 1025.

[19] V. Kambersky, Can. J. Phys. 48 (1970) 2906. 